\title{
ISOMETRIES AND DISCRETE ISOMETRY SUBGROUPS OF HYPERBOLIC SPACES
}

\author{
XI FU \\ Department of Mathematics, Hunan Normal University, \\ Changsha, Hunan 410081, People's Republic of China \\ and XIANTAO WANG* \\ Department of Mathematics, Hunan Normal University, \\ Changsha, Hunan 410081, People's Republic of China \\ e-mail:xtwang@hunnu.edu.cn
}

(Received 31 January 2007; accepted 6 October 2008)

\begin{abstract}
Let $\mathbb{M}^{n}$ be the $n$-dimensional hyperbolic space with $n \geq 2$. Suppose that $G$ is a discrete, sense-preserving subgroup of $I \operatorname{som} \mathbb{M}^{n}$, the isometry group of $\mathbb{\boxplus}^{n}$. Let $p$ be the projection map from $\mathbb{H}^{n}$ to the quotient space $M=\mathbb{H}^{n} / G$. The first goal of this paper is to prove that for any $a \in \partial \mathbb{M}^{n}$ (the sphere at infinity of $\mathbb{H}^{n}$ ), there exists an open neighbourhood $U$ of $a$ in $\mathbb{H}^{n} \cup \partial \mathbb{M}^{n}$ such that $p$ is an isometry on $U \cap \mathbb{H}^{n}$ if and only if $a \in{ }^{o} \Omega(G)$ (the domain of proper discontinuity of $G$ ). This is a generalization of the main result discussed in the work by Y. D. Kim (A theorem on discrete, torsion free subgroups of Isom $\mathbb{H}^{n}$, Geometriae Dedicata 109 (2004), 51-57). The second goal is to obtain a new characterization for the elements of $I s o m \mathbb{R} \mathbb{D}^{n}$ by using a class of hyperbolic geometric objects: hyperbolic isosceles right triangles. The proof is based on a geometric approach.
\end{abstract}

2000 Mathematics Subject Classification. Primary: 30F35, 51M10; Secondary: 20H10, 22E40.

1. Introduction. Let $n \geq 2, \mathbb{H}^{n}$ be the $n$-dimensional hyperbolic space and $\mathbb{B}^{n}$ be the Poincare ball model of $\mathbb{M}^{n}$, that is, $\mathbb{B}^{n}=\left\{x=\left(x_{1}, x_{2}, \ldots, x_{n}\right) \in \mathbb{R}^{n}:|x|<1\right\}$ with length differential $d s=\frac{2|d x|}{1-|x|^{2}}$. Let $\partial \mathbb{U}^{n}$ denote the sphere at infinity of $\mathfrak{H}^{n}$. We use $\mathbb{S}^{n-1}$ to denote $\partial \mathbb{B}^{n}$ and Isom $\llbracket \rrbracket^{n}$ the full group of the isometries of $\mathbb{\boxplus}^{n}$.

In this paper, $G$ always denotes a sense-preserving subgroup of $I \operatorname{som} \mathbb{U}^{n}$. The action of $G$ on $\mathbb{H}^{n}$ extends to a continuous action on the compactification of $\mathbb{U}^{n}$ by the sphere at infinity $\partial \mathbb{H}^{n}$. As in [6], let $\Lambda(G)$ and $\Omega(G)$ denote the limit set and the domain of discontinuity of $G$, respectively.

In [10, Section 12.1], the following is obtained:

Proposition 1.1. Suppose that $G$ is discrete and $a \in \Omega(G)$. Then there exists an open neighbourhood $U$ of a in $\mathbb{H}^{n} \cup \Omega(G)$ such that for each $f \in G$, either $U \cap f(U)=\emptyset$ or $U=f(U)$ and $f(a)=a$.

For $a \in \partial \mathbb{H}^{n}$, if there exists an open neighbourhood $U$ of $a$ in $\mathbb{M}^{n} \cup \partial \mathbb{H}^{n}$ such that for each non-trivial element $f \in G, U \cap f(U)=\emptyset$, then $a$ is called a properly discontinuous

*Corresponding author. 
point of $G$. The set of all properly discontinuous points of $G$, which is called the domain of proper discontinuity, is denoted by ${ }^{o} \Omega(G)$ (see [9] for the case $n=3$ ). It is obvious that ${ }^{o} \Omega(G) \subset \Omega(G)$ and $\Omega(G) \backslash{ }^{o} \Omega(G)$ consists of only fixed points of some elliptic elements of $G$. If $G$ is discrete and not finite, then $\Omega(G) \neq \varnothing$ if and only if ${ }^{o} \Omega(G) \neq \varnothing$. These imply following:

Proposition 1.2. If $G$ is discrete and torsion free, then ${ }^{\circ} \Omega(G)=\Omega(G)$.

Proposition 1.3. Suppose that $G$ is discrete and $a \in{ }^{\circ} \Omega(G)$. Then there exists an open neighbourhood $U$ of a in $\mathbb{H}^{n} \cup^{\circ} \Omega(G)$ such that for each non-trivial element $f \in G$, $U \cap f(U)=\emptyset$.

Let $p: \mathbb{H}^{n} \rightarrow M=\mathbb{H}^{n} / G$ be the projection map, where $G$ is discrete, $d_{\llbracket}$ be the hyperbolic metric of $\mathbb{R}^{n}$ and $d$ be defined on $M$ as follows:

$$
d(p(x), p(y))=\inf _{f \in G} d_{\mathbb{H}}(x, f(y)) \text { for } x, y \in \mathbb{H}^{n} .
$$

As the main result of [6], Kim proved the following:

THEOREM K. Suppose that $G$ is a discrete, torsion-free subgroup and $a \in \Omega(G)$. Then there exists an open neighbourhood $U$ of a in $\mathbb{\boxplus}^{n} \cup \Omega(G)$ such that

$$
d(p(x), p(y))=d_{\mathbb{\sharp}}(x, y) \quad \text { for } x, y \in U \cap \mathbb{H}^{n} .
$$

Firstly, we will prove the following:

THEOREM 1.4. Suppose that $G$ is a discrete subgroup. Then for any $a \in \partial \mathbb{U}^{n}$, there exists an open neighbourhood $U$ of a in $\llbracket^{n} \cup \partial \llbracket^{n}$ such that

$$
d(p(x), p(y))=d_{\mathbb{H}}(x, y) \text { for } x, y \in U \cap \mathbb{\boxplus}^{n}
$$

if and only if $a \in{ }^{o} \Omega(G)$.

As a corollary of Theorem 1.4 and Proposition 1.2, we can easily get the following:

COROLlARY 1.5. Suppose that $G$ is a discrete, torsion-free subgroup and $a \in \partial \mathbb{H}^{n}$. Then there exists an open neighbourhood $U$ of a in $\mathbb{H}^{n} \cup \partial \mathbb{U}^{n}$ such that

$$
d(p(x), p(y))=d_{\mathbb{H}}(x, y) \text { for } x, y \in U \cap \mathbb{\boxplus}^{n}
$$

if and only if $a \in \Omega(G)$.

REMARK 1.1. Corollary 1.5 shows that Theorem 1.4 is a generalization of Theorem $K$.

A map $f$ of $\mathbb{H}^{n}$ to itself is called r-hyperplane preserving if the image of any $r$-dimensional hyperplane in $\mathbb{W}^{n}$ under $f$ is still an $r$-dimensional hyperplane. When $r=1$, we call the corresponding map $f$ to be a geodesic-preserving map in $\mathbb{H}^{n}$. The relation between isometries and $r$-hyperplane preserving maps in $\mathbb{H}^{n}$ has been studied by many authors. For instance, in [5], Jeffers proved

THeOREM Je ([5, Theorem 3.6]). Suppose that $f: \mathbb{H}^{n} \rightarrow \mathbb{H}^{n}$ is a bijection. If $f$ is geodesic preserving, then $f$ is an isometry, i.e., $f \in I \operatorname{som} \mathbb{\boxplus}^{n}$. 
Recently, Li, Wang and Yao [7, 8] studied this relation too and obtained the following generalization:

Theorem LWY 1 ([7, Theorem 2] and [8, Theorem 3]). Suppose thatf : $\mathbb{H}^{n} \rightarrow \mathbb{H}^{n}$ is an r-hyperplane preserving map. Then $f$ is an isometry if and only iff is non-degenerate.

Here, $f$ is called degenerate if the image $f\left(\mathbb{H}^{n}\right)$ of $\mathbb{M}^{n}$ under $f$ is an $r$-hyperplane.

The second goal of this paper is to study this relation further. By using a class of hyperbolic geometric objects: hyperbolic isosceles right triangles, we get the following:

THEOREM 1.6. Suppose $f: \mathbb{H}^{n} \rightarrow \mathbb{H}^{n}$ is a continuous bijection. Then $f$ is an isometry in $\mathbb{\boxplus}^{n}$ if and only iff preserves hyperbolic isosceles right triangles in $\mathbb{U}^{n}$.

Here, we say that a map $f: \mathbb{H}^{n} \rightarrow \mathbb{H}^{n}$ preserves hyperbolic isosceles right triangles in $\mathbb{H}^{n}$ if for every hyperbolic isosceles right triangle in $\mathbb{H}^{n}$, its image under $f$ is still a hyperbolic isosceles right triangle in $\mathbb{H}^{n}$ and vertices correspond to vertices under $f$.

2. The proof of Theorem 1.4. For any non-trivial sense-preserving element $f \in$

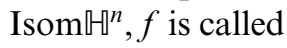

(1) elliptic if it has a fixed point in $\mathbb{H}^{n}$;

(2) parabolic if it has only one fixed point in $\partial \mathbb{H}^{n}$ and none in $\mathbb{H}^{n}$;

(3) loxodromic if it has two fixed points in $\partial \mathbb{H}^{n}$ and none in $\mathbb{H}^{n}$.

Suppose $f$ is loxodromic and its fixed points are $x$ and $y$. We say that $x$ is attractive if $f^{r}(z) \rightarrow x$ as $r \rightarrow+\infty$ for any $z \in \partial \mathbb{Q}^{n}-\{y\}$. And $y$ is called repulsive (cf. [4]). Then $y$ is the attractive fixed point of $f^{-1}$ and $x$ the repulsive one.

2.1. Preliminary lemmas. As in [14], let $\Gamma_{n}$ denote the $n$-dimensional Clifford group; see $[\mathbf{1}, \mathbf{2}, \mathbf{1 2}-\mathbf{1 4}, \mathbf{1 6}]$ etc. for the representation of sense-preserving Möbius transformations by using the Clifford numbers in $\Gamma_{n}$ and its applications. It easily follows from [1, Theorem A] or [2, Vahlen's theorem] that

LEMMA 2.1. Every sense-preserving element $f$ in Isom $\mathbb{B}^{n}$ has the following representation:

$$
f=\left(\begin{array}{cc}
a & b \\
b^{\prime} & a^{\prime}
\end{array}\right),
$$

where $a, b \in \Gamma_{n-1} \cup\{0\}$, $a b^{*}, \bar{a} b \in \overline{\mathbb{R}}^{n-1}$ and $|a|^{2}-|b|^{2}=1$.

Let $f=\left(\begin{array}{cc}a & b \\ b^{\prime} & a^{\prime}\end{array}\right) \in I \operatorname{som} \mathbb{B}^{n}$ be sense preserving and $b \neq 0$, i.e., $f(\infty) \neq \infty$. Then

$$
S\left(c_{f}, r_{f}\right)=\left\{x \in \mathbb{S}^{n-1}:\left|x-\left(b^{\prime}\right)^{-1} a^{\prime}\right|=\frac{1}{|b|}\right\}
$$

is called the isometric sphere of $f$, where $c_{f}=\left(b^{\prime}\right)^{-1} a^{\prime}$ and $r_{f}=\frac{1}{|b|}$ are the centre and the radius of $S\left(c_{f}, r_{f}\right)$, respectively.

For any $z \in \mathbb{H}^{n} \cup \partial \mathbb{W}^{n}$, let

$$
\operatorname{Stab}_{G}(z)=\{g \in G: g(z)=z\},
$$

which is called the stabilizer of $z$ in $G$. 
By using Lemma 2.1, we can get the following generalization of [6, Proposition 1]:

Lemma 2.2. Suppose that $G$ is a discrete subgroup of Isom $\mathbb{B}^{n}$ and $G \backslash \operatorname{Stab}_{G}(O) \neq$ $\emptyset$, where $O$ denotes the origin of $\mathbb{B}^{n}$. For $f \in G \backslash \operatorname{Stab}_{G}(O)$, let $f=A_{f} \circ i_{f}$ be the decomposition of $f$ as in [6, Theorem 3], where $i_{f}$ is the reflection in the sphere $S\left(c_{f}, r_{f}\right)$ (cf. [3]). Then

$$
\sup _{f \in G \backslash \operatorname{Stab}_{G}(O)} r_{f}<\infty
$$

Proof. Suppose

$$
\sup _{f \in G \backslash \operatorname{Stab}_{G}(O)} r_{f}=\infty .
$$

Then there is an infinite sequence $\left\{f_{m}\right\}$ in $G$ such that

$$
r_{f_{m}} \rightarrow \infty .
$$

By Lemma 2.1, we may assume that

$$
f_{m}=\left(\begin{array}{ll}
a_{m} & b_{m} \\
b_{m}^{\prime} & a_{m}^{\prime}
\end{array}\right)
$$

where $b_{m} \neq 0$.

Then

$$
\left|b_{m}\right|^{-1}=r_{f_{m}} \rightarrow \infty
$$

This yields

$$
b_{m} \rightarrow 0 \text { and }\left|a_{m}\right| \rightarrow 1
$$

since $\left|a_{m}\right|^{2}-\left|b_{m}\right|^{2}=1$.

It follows from

$$
f_{m}(O)=\frac{a_{m} b_{m}^{*}}{\left|a_{m}\right|^{2}}
$$

that

$$
f_{m}(O) \rightarrow O \text { as } m \rightarrow \infty .
$$

This implies that $O \in \Lambda(G) \subset \mathbb{S}^{n-1}$. This is the desired contradiction.

We recall the following result from [10].

Lemma 2.3 [10, Theorem 5.5.1]. If $G$ is discrete and purely elliptic (that is, each non-trivial element of $G$ is elliptic), then there exists $\eta \in \mathbb{H}^{n}$ such that $f(\eta)=\eta$ for each $f \in G$.

REMARK 2.1. The condition ' $G$ being discrete' in Lemma 2.3 cannot be removed (cf. [15]).

Lemma 2.4. Suppose that $G$ is discrete. For any $a \in \partial \mathbb{Q}^{n}$, if there exists an open neighbourhood $U$ of a in $\mathbb{\boxplus}^{n} \cup \partial \mathbb{Q}^{n}$ such that

$$
d(p(x), p(y))=d_{\sharp}(x, y) \text { for } x, y \in U \cap \mathbb{\boxplus}^{n},
$$

then $U \cap \partial \mathbb{Q}^{n} \subset \Omega(G)$. In particular, $a \in \Omega(G)$. 
Proof. Suppose that there exists some $b \in \Lambda(G) \cap\left(U \cap \partial \mathbb{\boxplus}^{n}\right)$, for the contradiction. Since loxodromic fixed points are dense in the limit set (see, for example, [11, Theorem B1] or [3, Theorem 5.3.8]), we may assume that $b$ is fixed by some $f \in G$ which is loxodromic or parabolic. Without loss of generality, we assume that $b$ is the attractive fixed point of $f$ if $f$ is loxodromic. For any $x \in U \cap \mathbb{H}^{n}$, there exists a sufficiently large number $r>0$ such that $f^{r}(x) \in U \cap \mathbb{H}^{n}$ and $f^{r}(x) \neq x$.

Let $y=f^{r}(x)$. Then

$$
d(p(x), p(y))=\inf _{g \in G} d_{\llbracket}(x, g(y)) \leq d_{\llbracket}\left(x, f^{-r}(y)\right)=0<d_{\llbracket}(x, y) .
$$

This is the desired contradiction.

2.2. The proof of Theorem 1.4. In the proof, we use the Poincaré ball model $\mathbb{B}^{n}$ of $\dddot{H}^{n}$.

Since $d_{E}$ (the topological Euclidean metric on $\mathbb{B}^{n}$ ) is invariant under the subgroup $\operatorname{Stab}_{G}(O)$, it implies that, except for Theorem 2 and Proposition 1, all other theorems, propositions and lemmas used in the proof of [6, Theorem 1] (i.e., Theorem $K$ ) also hold in the case of $G$ being only discrete. Hence, the proof of the sufficiency follows from Proposition 1.3, Lemma 2.2 and similar discussions as those in [6].

Here, we prove the necessity.

Since the assumptions in Lemma 2.4 are satisfied it follows that $a \in \Omega(G)$. Suppose $a \notin^{o} \Omega(G)$, for the contradiction. Then there exists some elliptic element $h \in G$ such that $h(a)=a$. Then $\operatorname{Stab}_{G}(a)$ is non-trivial and purely elliptic. It follows from Lemma 2.3 that there is $\eta \in \mathbb{B}^{n}$ such that

$$
g(\eta)=\eta \quad \text { for any } g \in \operatorname{Stab}_{G}(a)
$$

Let $A$ be the hyperbolic geodesic in $\mathbb{B}^{n}$ with the endpoint $a$ passing through $\eta$. Let $\omega \in \mathbb{S}^{n-1}$ be the other endpoint of $A$. Then $\omega$ is also fixed by each element of $\operatorname{Stab}_{G}(a)$. This implies that there exists a neighbourhood $V \subset \mathbb{S}^{n-1} \cup \mathbb{B}^{n}$ of $a$ such that

$$
V \subset U \text { and } g(V)=V \text { for every } g \in \operatorname{Stab}_{G}(a) .
$$

We can find $x \in V \cap \mathbb{B}^{n}$ and $g \in \operatorname{Stab}_{G}(a)$ such that $g(x) \neq x$. Let $y=g(x)$. Then $y \in V$ and

$$
d_{\mathbb{B}}(x, y)>0,
$$

but

$$
d(p(x), p(y))=0
$$

This contradiction completes the proof.

3. The proof of Theorem 1.6. Here, we also use the Poincaré ball model $\mathbb{B}^{n}$ of $\mathbb{Q}^{n}$. We always use $A, B, C, \ldots$ to denote the points in $\mathbb{B}^{n}$. Also we denote by $A^{\prime}, B^{\prime}$, $C^{\prime}, \ldots$ the images of $A, B, C, \ldots$ under $f$, by $\widehat{A B}$ the geodesic segment between $A$ and $B$, by $\triangle A B C$ the hyperbolic triangle with vertices $A, B$ and $C$, and by $\angle A B C$ the angle between $\widehat{A B}$ and $\widehat{B C}$. Recall that $O$ denotes the origin of $\mathbb{B}^{n}$. 
Here, we assume that $f: \mathbb{B}^{n} \rightarrow \mathbb{B}^{n}$ is a continuous bijection that preserves the hyperbolic isosceles right triangles in $\mathbb{B}^{n}$ and fixes the origin $O$. For any hyperbolic triangle $A O B$, we use $\mathbb{B}_{\triangle A O B}^{2}$ to denote the intersection of the two-dimensional hyperplane in $\overline{\mathbb{R}}^{n}$ containing $\triangle A O B$ and $\mathbb{B}^{n}$, which is a two-dimensional unit disk with the centre $O$.

\subsection{Preliminary lemmas.}

LEMMA 3.1. For any hyperbolic isosceles right triangle, it is uniquely determined by its acute angle.

Proof. It easily follows from [3, Theorem 7.11.2].

LEMMA 3.2. Suppose $\triangle A O B$ is a hyperbolic isosceles right triangle in $\mathbb{B}^{n}$ and $\angle A O B$ is the right angle. Then $\angle A^{\prime} O^{\prime} B^{\prime}$ is the right angle in $\triangle A^{\prime} O^{\prime} B^{\prime}$.

Proof. Assume the contradiction. Without loss of generality, we may assume that $\angle O^{\prime} A^{\prime} B^{\prime}$ is the right angle in $\triangle O^{\prime} A^{\prime} B^{\prime}$. We may find a point $C \in \mathbb{B}_{\triangle A O B}^{2}$ which satisfies that $d_{\mathbb{B}}(O, A)=d_{\mathbb{B}}(A, C), \angle O A C$ is a right angle and $\widehat{O C}$ intersects $\widehat{A B}$ with the intersection point $D$. Then $D$ lies in the interior of $\widehat{A B}$ and $\triangle O A C$ is a hyperbolic isosceles right triangle. Since $f$ is a bijection and preserves hyperbolic isosceles right triangles, we see that $\triangle O^{\prime} A^{\prime} C^{\prime}$ is also a hyperbolic isosceles right triangle and $D^{\prime}$ is an interior point of $\widehat{A^{\prime} B^{\prime}}$. Obviously, $\angle O^{\prime} A^{\prime} C^{\prime}>\frac{\pi}{2}$. It follows from [3, Theorem 7.16.2] that this is a contradiction.

LEMMA 3.3. Suppose $\triangle A O B$ is a hyperbolic isosceles right triangle in $\mathbb{B}^{n}$ and $\angle A O B$ is an acute angle. Then $\angle A^{\prime} O^{\prime} B^{\prime}$ is also an acute angle.

Proof. Assume the contradiction. Then $\angle A^{\prime} O^{\prime} B^{\prime}$ is the right angle in $\triangle A^{\prime} O^{\prime} B^{\prime}$. We may find a point $C$ in $\mathbb{B}_{\triangle A O B}^{2}$ such that $\triangle A O C$ is a hyperbolic isosceles right triangle with $\angle A O C$ being the right angle and $\widehat{O B}$ intersects $\widehat{A C}$ with the intersection point $D$. Then $\triangle A^{\prime} O^{\prime} C^{\prime}>\frac{\pi}{2}$ and $D^{\prime}$ is an interior point of $\widehat{O^{\prime} B^{\prime}}$. This is a contradiction by [3, Theorem 7.16.2].

Lemma 3.4. Suppose $\triangle A O B$ is a hyperbolic isosceles right triangle with $\angle O A B$ being the right angle. Then $\angle O^{\prime} A^{\prime} B^{\prime}$ is a right angle.

Proof. Assume the contradiction. By Lemma 3.3, we know that $\angle A^{\prime} O^{\prime} B^{\prime}$ is an acute angle. Hence, $\angle O^{\prime} B^{\prime} A^{\prime}$ is the right angle. Choose two points $D$ and $E$ in the interior of $\widehat{O A}$ and $\widehat{A B}$, respectively, such that $d_{\mathbb{B}}(D, A)=d_{\mathbb{B}}(A, E)$. Then $\triangle D^{\prime} A^{\prime} E^{\prime}$ is a hyperbolic isosceles right triangle. Obviously, $D^{\prime}$ and $E^{\prime}$ are interior points in $\widehat{A^{\prime} O^{\prime}}$ and $\widehat{A^{\prime} B^{\prime}}$, respectively. By Lemma 3.1, this is the desired contradiction.

LEMMA 3.5. $f$ preserves any angle with the vertex origin $O$.

Proof. Let $\angle A O B$ be any angle in $\mathbb{B}^{n}$. We come to prove that $\angle A O B$ is the same as $\angle A^{\prime} O^{\prime} B^{\prime}$. By Lemma 3.2 and the hypothesis $f$ being a bijection, we may assume that $\angle A O B$ is an acute angle. Let us start our discussions with the following special cases.

Case I. $\angle A O B=\frac{\pi}{p}$ with $p>4$. 
By [3, Theorem 7.16.2], we may assume that $\triangle A O B$ is a hyperbolic isosceles right triangle with the angle $\angle O A B$ being right angle. In $\mathbb{B}_{\triangle A O B}^{2}$, let

$$
\begin{aligned}
& K_{1}=\left\{z \in \mathbb{B}_{\triangle A O B}^{2}: d_{\mathbb{B}}(O, z)=d_{\mathbb{B}}(O, A)\right\}, \\
& K_{2}=\left\{z \in \mathbb{B}_{\triangle A O B}^{2}: d_{\mathbb{B}}(O, z)=d_{\mathbb{B}}(O, B)\right\}
\end{aligned}
$$

and the rays $r_{i}(i=1,2, \ldots, 2 p)$ from $O$ satisfy that the $2 p$ rays $r_{i}$ are anticlockwise arranged from $r_{1}$ to $r_{2 p}$ and each angle formed by $r_{i}$ and $r_{i+1}$ is $\frac{\pi}{p}$, where we assume that $A$ lies in $r_{1}$ and $B$ in $r_{2}$.

We also let $A_{i}$ be the intersection point of $K_{1}$ and $r_{i}$, and $B_{i}$ the one of $K_{2}$ and $r_{i}$, where $i=1,2, \ldots, 2 p, A_{1}=A$ and $B_{2}=B$.

Then each hyperbolic triangle $\triangle A_{i} O B_{i+1}$ is an isosceles right one $(i=1,2, \ldots, 2 p)$, where $B_{2 p+1}=B_{1}$, and the union of the closures of all $\Delta A_{i} O B_{i+1}(i=1,2, \ldots, 2 p)$ consists of a neighbourhood of $O$. By Lemmas 3.3 and 3.4, and the hypothesis $f$ being a bijection, we know that $\angle A^{\prime} O^{\prime} B^{\prime}=\angle A O B=\frac{\pi}{p}$.

Case II. $\angle A O B=\frac{\pi}{3}$.

By dividing $\angle A O B$ into two $\frac{\pi}{6}$-valued angles and Case I, we see that $\angle A^{\prime} O^{\prime} B^{\prime}=$ $\angle A O B$.

Case III. $\angle A O B=\frac{\pi}{4}$.

Similar discussions as in Case II show that $\angle A^{\prime} O^{\prime} B^{\prime}=\frac{\pi}{4}$.

Case IV. $\angle A O B=\frac{q \pi}{p}$, where the two natural numbers $p$ and $q$ are prime.

Since $\angle A O B$ is acute, we see that $0<2 q<p$. By the discussions as mentioned above, we may assume that $p>4$. Let us divide $\angle A O B$ into $q^{2}$ many $\frac{\pi}{p q}$-valued angles. Then it follows from Case I that $\angle A^{\prime} O^{\prime} B^{\prime}=\angle A O B$.

For general case, since $f$ is continuous, it follows from Case IV that $\angle A^{\prime} O^{\prime} B^{\prime}=$ $\angle A O B$. The proof is complete.

3.2. The proof of Theorem 1.6. The necessity is obvious. Hence, we only need to prove the sufficiency.

By composite with some element in $I \operatorname{som} \mathbb{B}^{n}$, we may assume that $f$ fixes $O$. Let $A$ be an arbitrary point in $\mathbb{B}^{n}$ which is different from $O$. Then we can find a hyperbolic isosceles right triangle $\triangle A O B$ such that $\widehat{O A}$ is a side of $\triangle A O B$ and $\angle A O B$ is an acute angle. It follows from Lemmas 3.1, 3.2 and 3.5 that $d_{\mathbb{B}}(O, A)=d_{\mathbb{B}}\left(O^{\prime}, A^{\prime}\right)$. Then for any points $B$ and $C$ in $\mathbb{B}^{n}$, we see that $d_{\mathbb{B}}\left(O^{\prime}, B^{\prime}\right)=d_{\mathbb{B}}(O, B), d_{\mathbb{B}}\left(O^{\prime}, C^{\prime}\right)=d_{\mathbb{B}}(O, C)$ and by Lemma 3.5, we also see that $\angle A^{\prime} O^{\prime} B^{\prime}=\angle A O B$. These imply that $d_{\mathbb{B}}\left(B^{\prime}, C^{\prime}\right)=$ $d_{\mathbb{B}}(B, C)$. These mean that $f$ is an isometry. This completes our proof.

ACKNOWLEDGEMENTS. The authors would like to thank the referee for the careful reading of this paper and the useful suggestions. The research was partly supported by program for NCET (No. 04-0783), NSFs of China (No. 10771059) and Hunan (No. 05JJ10001).

\section{REFERENCES}

1. L. V. Ahlfors, Möbius transformations and Clifford numbers, in Differential geometry and complex analysis (Chavel I. and Farkas H. M., Editors) (Springer-Verlag, Berlin, 1985), $65-73$. 
2. L. V. Ahlfors, On the fixed points of Möbius transformations in $\bar{R}^{n}$, Ann. Acad. Sci. Fen. Ser. A. I. Math. 10 (1985), 15-27.

3. A. F. Beardon, The geometry of discrete groups, Graduate text in mathematics, vol. 91 (Springer-Verlag, New York, 1983).

4. F. W. Gehring and G. J. Martin, Discrete quasiconformal groups, Proc. Lond. Math. Soc. 55 (1987), 331-385.

5. J. Jeffers, Lost theorems of geometry, Am. Math. Monthly 107 (2000), 800-812.

6. Y. D. Kim, A theorem on discrete, torsion free subgroups of Isom! $\rrbracket^{n}$, Geom. Dedicata 109 (2004), 51-57.

7. B. Li and Y. Wang, Transformations and non-degenerate maps, Sci. China 48 (2005), $195-205$.

8. B. Li amd G. Yao, On characterizations of sphere-preserving maps, Mathematical Proceedings of Cambridge Philosophical Society, to appear.

9. B. Maskit, Kleinian groups (Springer-Verlag, New York, 1988).

10. J. G. Ratcliffe, Foundations of hyperbolic manifolds, Graduate text in mathematics, Vol. 149 (Springer-Verlag, New York, 1994). 578.

11. P. Tukia, Differentiability and rigidity of Möbius groups, Invent. Math. 2 (1985), 557-

12. X. Wang and L. Li, Algebraic convergence theorems of $n$-dimensional Kleinian groups, Israel J. Math. 162 (2007), 221-233.

13. X. Wang, L. Li and W. Cao, Discreteness criteria for Möbius groups acting on $\bar{R}^{n}$, Israel J. Math. 150 (2005), 357-368.

14. X. Wang and W. Yang, Discreteness criteria of Möbius groups of high dimensions and convergence theorem of Kleinian groups, Adv. Math. 159 (2001), 68-82.

15. P. L. Waterman, Purely elliptic Möbius groups, in Holomorphic functions and moduli, vol. II (Springer, New York, 1983), 173-178.

16. P. Waterman, Möbius transformations in several dimensions, Adv. Math. 101 (1993), $87-113$. 